\title{
Exploring Multitemporal Hydro Power Models of the Nordic Power System using Spine Toolbox
}

\author{
Iasonas Kouveliotis-Lysikatos, Manuel Marin, Mikael Amelin and Lennart Söder \\ School of Electrical Engineering and Computer Science \\ KTH Royal Institute of Technology, Stockholm, Sweden, SE-100 44 \\ Email: \{iasonas, manuelma, amelin, lsod\}@kth.se
}

\begin{abstract}
This paper presents the development and simulation of hydro power models in the open source energy modelling framework Spine. We study the market-based hourly operation of the Skellefte river in the Swedish hydro power system using the abstract representation of Spine Model, in order to employ and demonstrate its available functionalities, focusing on the automated handling of the temporal resolution of the optimization model. The Spine temporal block is used for automating the transformation of the temporal resolution of the model in different time intervals, as well as the manipulation of various modelling parameters. After the mathematical formulation of the optimization problem and the detailed analysis of the modelling steps in Spine, various results are extracted discussing the added value of Spine.
\end{abstract}

Index Terms-Spine, temporal block, hydro power modelling, Nordic System, Skelefte river.

\section{NOMENCLATURE}

\section{Parameters}

$\lambda_{t} \quad$ Expected spot price for time $t$.

$\overline{M_{i}} \quad$ Maximum capacity of reservoir $i$.

$\overline{Q_{i, j}} \quad$ Maximum water discharge for power plant $i$ and line segment $j$.

$\overline{S_{i}} \quad$ Maximum water spillage of power plant $i$.

$\rho \quad$ Penalty for discharge oscillations.

$\tau_{i}^{q} \quad$ Time delay for the discharged water of power plant $i$ to reach the downstream power plant.

$\tau_{i}^{s} \quad$ Time delay for the spilled water of power plant $i$ to reach the downstream power plant.

$Q_{i, j} \quad$ Minimum water discharge for power plant $i$ and line segment $j$.

$S_{i} \quad$ Minimum water spillage of power plant $i$.

$\bar{Q}_{i}^{a v g} \quad$ Yearly average inflow of water to each reservoir per hour.

$V_{i, t} \quad$ Water inflow for reservoir $i$ and time $t$.

\section{Indices and Sets}

$A_{i}^{Q} \quad$ Set of upstream stations for station $i$ (discharge).

$A_{i}^{S} \quad$ Set of upstream stations for station $i$ (spillage).

$I \quad$ Set of reservoirs and hydro power plants.

$J \quad$ Set of line segments of the water discharge functions.

$T \quad$ Set of time steps.

\section{Variables}

$\delta_{i, t}^{+} \quad$ Positive increase of discharge.

$\delta_{i, t}^{-} \quad$ Positive decrease of discharge.

This project has received funding from the European Union's Horizon 2020 research and innovation programme under grant agreement N. 774629. $\mu_{i, j} \quad$ Marginal production equivalent for power plant $i$ and line segment $j$.

$M_{i, t} \quad$ Water content of reservoir $i$ at the end of hour $t$.

$P_{t} \quad$ Total power production for time $t$.

$Q_{i, j, t} \quad$ Water discharge for power plant $i$, line segment $j$ and time $t$.

$Q_{i, t} \quad$ Total water discharge for power plant $i$ and time $t$.

$S_{i, t} \quad$ Water spillage of power plant $i$ and time $t$.

\section{INTRODUCTION}

The efficient operation of large hydrologically coupled hydro power plants within competitive electricity markets is a challenging problem for which many different methodologies and tools have been developed over the past years [1]. In particular, novel, efficient techniques for calculating the optimal discharge schedule of complex hydro power plant systems with reservoirs (either for short-term or long-term horizons) are actively being studied. That need has become ever more important nowadays due to their beneficial balancing capabilities, as intermittent Renewable Energy Systems are increasingly replacing conventional nuclear and fossil fuelbased systems [2].

From the energy modelling software perspective, various solutions currently exist, either for energy systems in general, or for specialized modelling of hydro power systems. Apart from their specialization and modelling capabilities though, another important aspect (specifically for the academic community) is whether they are open source or not. The Balmorel energy system model is an example of an open source generic energy model [3] that has been used for hydro power applications, but still relies on the GAMS software that is not open source. The EMPS - multi area power-market simulator, includes a detailed representation of hydro power models as a main functionality [4], but is not open source. In [5], the author reviews and compares in high detail the previous and other solutions for hydro power modelling.

However, in order to open up simulation of hydro power systems for a broader use, there is a value of using opensource software for the simulation. In this paper we showcase the capabilities of the open source energy modelling software Spine. Even though Spine begins from a generic energy flow-based model, eventually it provides the capability to construct hydro power models with an easily customizable level of detail, as a way to balance between accuracy and 
computational complexity. Specifically, it is demonstrated how a rather detailed hydro power model can be developed from simple building blocks in a generic energy system model like Spine. As a next step, it is investigated how the temporal resolution influences the accuracy and the computation time of the developed hydro power model by comparing the results from a high time resolution model with lower time resolution instances, as well as a case where the water delay time between hydro power plants is ignored.

\section{Modelling with Spine Toolbox}

\section{A. Spine Model and Spine Toolbox}

Spine is an open source generic energy modelling framework [6]. It consists of two main components, the Spine Toolbox and the Spine Model. The Spine Toolbox enables the definition and management of various energy systems models, including Spine Model itself, which is a dedicated tool for multi-vector energy system modelling. In that way, Spine Toolbox encompasses Spine Model and a set of ancillary tools for data gathering, representation, persistent storage and visualization, and orchestrates the execution and validation of complex scenarios.

The Spine data structure sets the ground for conceptualizing generic multi-energy models. In combination with the data importing and exporting tools, the user is able to define specialized recipes for passing the data between Spine and other applications such as GAMS, or MATLAB. Software and user interfaces enable the integration of the various components and facilitate the model construction and validation by the end-user. The Spine toolbox is implemented in Python, while Spine Model is implemented in Julia. A high-level diagram of the Spine software suite is provided in Fig. 1.

As shown in Fig. 1, Spine spreads over two execution environments, appropriately interlinking them, and providing the necessary software interfaces for accommodating custom (even proprietary) software modules. The Spine model, with its temporal structure represented by the temporal block object (Fig. 1 left), is executed in a Julia environment, while the other core Spine functionalities and services such as the Spine data store, and the tools for the definition, parameterization, and visual (object-oriented) representation of the model live in a Python execution environment (Fig. 1 right). Spine is bundled with a friendly user interface developed in the crossplatform widget toolkit Qt, accessible in Python via the PySide2 module.

\section{B. The Spine Model Building Blocks}

The Spine data structure is based on a generic Entity - Attribute - Value basis, using Classes and Relationships (in short EAV/CR), tailored to represent multi-vector energy systems. The abstract classes and the relationships between them enable the formulation of a multi-commodity, flow-based model of an energy system, in an object-oriented manner. The main abstract classes are described below [7]:

- Node: a point in space where energy from a certain vector gets balanced.

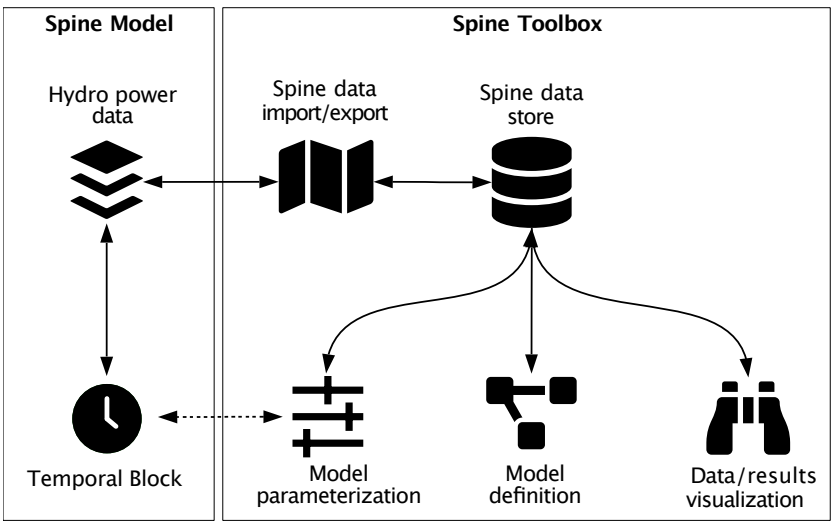

Fig. 1. Spine conceptual diagram and the temporal block component.

- Unit: a device, installed between two nodes, capable of converting energy from one vector to another.

- Connection: a device, also installed between two nodes, capable of transferring energy within the same vector, or enabling the flow of commodity.

- Commodity: a type of energy flow, uniquely associated with a vector.

- Temporal block: a period of time with a specific resolution.

For representing the topology of a commodity flow model, the modeler uses the available building blocks described above, i.e. by instantiating the respective abstract classes and defining relationships according to the topological characteristics of the actual system and the coupling constraints between the nodal variables and flow variables. This process is handled by the user interface of Spine toolbox (e.g. by draggingand-dropping icons representing the data classes) and do not require a direct intervention in lower level code. Depending on the needs of the application, various parameters (predefined or even custom) can be specified as a way to capture the physics of the flow of each commodity and the transformations between them.

When the implementation of the model is completed and all the parameters are appropriately set, the modeler can execute it and retrieve the results. The optimization model, i.e. the Spine Model, is based on the powerful JuMP modelling language for Julia [8]. The SpineModel.jl package handles the automated building and execution of the optimization model (variables, constraints and objective function), essentially decoupling the modeler from providing any lines of Julia code unless that is desired. In the latter case, the modeler can access any JuMP or SpineModel methods directly from the Julia command line, conveniently integrated into the Spine Toolbox user interface.

\section{Temporal Blocks in Spine}

The Spine temporal block object class enables the manipulation of the temporal resolution of the energy models in an automated way. Its purpose is twofold, firstly to provide the user with the capability of compiling abstract models based on 
time series data sets that have different resolutions for different components of the model, and secondly, to enable the user to effortlessly modify the time resolution of the whole model (or specific parts of it) for different executions of simulation scenarios [9]. A typical use-case for multiple temporal blocks would be the simulation of an energy model with multiple commodities, some of which are tracked in a lower detail than others. For example, in a fuel-based power plant the dynamics of the variables modelling the fuel consumption could possibly be more efficiently captured using a 3-hour time resolution, while the variables of the electricity flow would need to be modeled in an hourly basis (for evaluating the interaction with the day ahead electricity market). In practice, starting from the time resolution of the available data, Spine allows the modeler to specify the time period that the decision variable should represent. In addition to that, when a decision variable is chosen to be scaled upwards in time (from the resolution of the available data), it is possible to select the function aggregating the corresponding subset data of the time series, from a number of alternatives such as: the mean value, the maximum or minimum, or the evaluation of some custom function.

\section{HYDRo POWER MODELLING}

\section{A. Problem formulation}

The optimization problem for the optimal hydro power plants operation is presented next. The objective (1a) seeks to maximize the revenues from the power that is generated [10], minus a penalty term that aims at discouraging the swift changes in the discharge vector of each power plant, thus providing smoother solutions [11]:

$$
\max \sum_{t=1}^{T} \lambda_{t} P_{t}-\rho_{i} \cdot \sum_{i=1}^{I}\left(\delta_{i, t}^{+}+\delta_{i, t}^{-}\right)
$$

subject to:

$$
\begin{gathered}
M_{i, t}=M_{i, t-1}+V_{i, t}-\sum_{j \in J} Q_{i, j, t}-S_{i, t} \\
+\sum_{k \in A_{i}^{Q}}\left(\sum_{j \in J} Q_{k, j, t-\tau_{i}^{q}}\right)+\sum_{k \in A_{i}^{S}} S_{k, t-\tau_{i}^{s}}, \\
P_{t}=\sum_{i \in I} \sum_{j \in J} \mu_{i, j} Q_{i, j, t}, \\
\overline{Q_{i, j}} \geqslant Q_{i, j, t} \geqslant \underline{Q_{i, j}} \\
\overline{M_{i}} \geqslant M_{i, t} \geqslant \underline{M_{i}}, \\
\overline{S_{i}} \geqslant S_{i, t} \geqslant \underline{S_{i}} \\
\delta_{i, t}^{+}-\delta_{i, t}^{-}=Q_{i, t}-Q_{i, t-1}, \\
\delta_{i, t}^{+}, \delta_{i, t}^{-} \geqslant 0, \\
\forall i \in I, j \in J, t \in T
\end{gathered}
$$

Constraint (1b) imposes the so-called hydrological balance for each node (power plant), while the expression (1c) represents the total power generated from the water discharged by each power plant taking into account the different efficiency line segments. Constraints (1d) - (1h), enforce the various technical limits and express the positive rate of change of the discharge. For the effective modelling of the initial phase of the model, that is $t \leq \tau_{i}^{q}$ for each plant, the hydrological balance (1b) is redefined at a higher lever of detail as follows:

$$
\begin{array}{r}
M_{i, t}=M_{i, t-1}+V_{i, t}-Q_{i, t}-S_{i, t} \\
+\sum_{k \in A_{i}^{Q}} Q_{k, t-\tau_{i}^{q}}+\sum_{k \in A_{i}^{S}} S_{k, t-\tau_{i}^{s}} \\
+\left.\sum_{k \in A_{i}^{Q}} Q_{k}^{a v g}\right|_{t \leq \tau_{k}^{q}}+\left.\sum_{k \in A_{i}^{Q}} \frac{60-m_{k}^{q}}{60} Q_{k}^{a v g}\right|_{t=\tau_{k}^{q}+1} \\
Q_{i, t-\tau_{i}^{q}}=\frac{m_{i}^{q}}{60} Q_{i, t-h_{i}^{q}}+\frac{60-m_{i}^{q}}{60} Q_{i, t-h_{i}^{q}-1}
\end{array}
$$

Assuming that the initial and final reservoir contents are inputs to the problem, the above expressions are used to estimate the power production of the plants for the first hours until water from the upstream stations reaches all plants. Equation (2) constitutes a practical way of modelling the water flow delays between succeeding power plants, handling respectively the initialization of the model. Assuming also a constant time delay for each plant, independent of the discharge, the quantity $\tau_{i}^{q}$ can be expressed in hours $\left(h_{i}^{q}\right)$ and minutes $\left(m_{i}^{q}\right)$. Equation (3) provides then the delayed upstream flow as a weighted average of the discharge between the hours $h j$ and $h j+1$, as described in [12]. A similar expression is used for the spillage delays.

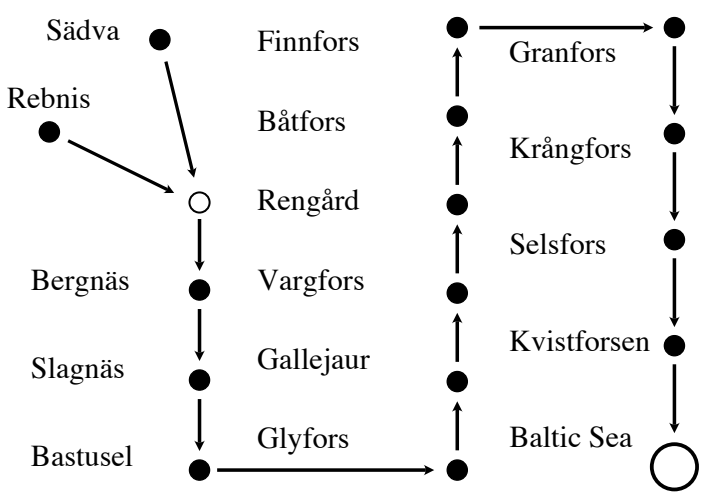

Fig. 2. The Skellefte river flow routes.

\section{B. The Skellefte Hydro Power System}

The hydro power system of the Skellefte river in the Swedish hydro power system includes fifteen power stations, whose topology is shown in Fig. 2. The hydro power model aims at finding the optimal solution for the operation of all 


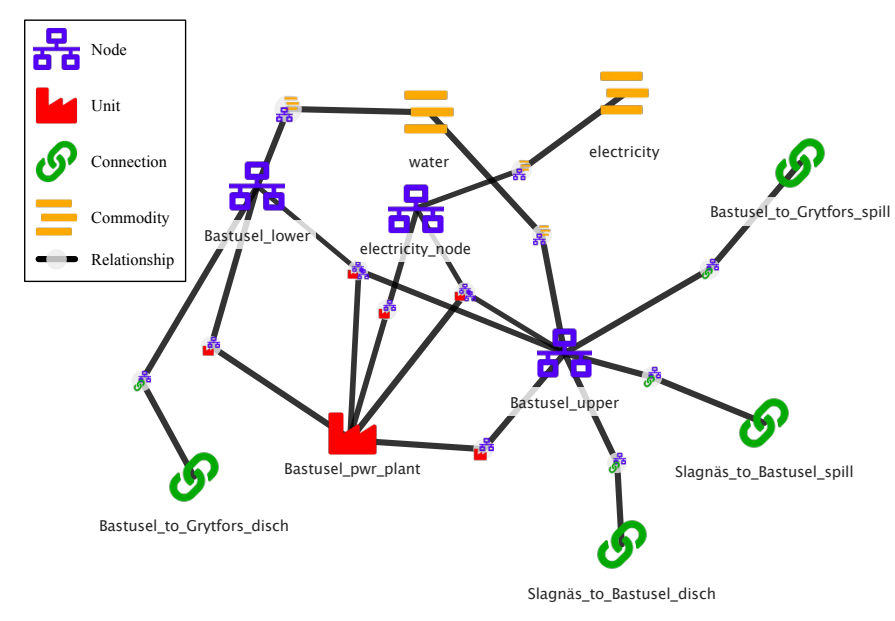

Fig. 3. Illustrative example of modelling a single hydro power plant in Spine, the Bastusel plant.

power plants taking into account the size of their reservoirs as well as the flow delays for the water that is spilled and discharged. Following the formulation of (1a), the optimal solution targets the maximization of the revenues from the produced power for a period of one week. The input data comprise the hourly electricity prices for the week under consideration, and the following parameters for each power station:

- The capacity of each station, i.e., maximum electricity output.

- The conversion curve from water to electricity. For this study, a simple piecewise-linear curve with two segments is used. The conversion ratio of the second segment is lower than the first one, avoiding thus a mixed integer formulation [13].

- The initial, final, and maximum reservoir levels.

- The minimum (and maximum) hourly water discharge and spillage.

- The topology (as it is shown in Fig. 2) of the power stations and the water flow delays for both spillage and discharge of each plant. Note that the topology of the water flow for spillage and discharge coincide in our case.

- The hourly local inflow, at each power plant.

- The average hourly water discharge.

Some simplifying assumptions are taken for the development of the hydro power model. Firstly, all power plants are assumed to have been discharging the average amount of water before the beginning of the week and secondly, the electricity grid is not taken into consideration.

\section{Implementing the Skellefte Hydro Power System in Spine}

The implementation of an optimization problem in Spine Model is performed by using the available Spine building blocks (presented in subsection II-B). The modeller can introduce in addition custom expressions if that is required, but normally the generality of Spine Model should be able to accommodate flow-based optimization models of a high diversity. The first step for expressing constraints (1b) - (1h) under the conventions of Spine Model, is to identify the nodes of the Spine data structure. Keeping in mind that the nodes have essentially the capability to impose the balance of a certain commodity flowing in and out of them, the nodes can be set to represent the hydrological balance constraints (1b), while the unit data structure can be used for representing the power plant, by setting the conversion rates $\left(\mu_{i, j}\right)$, for modelling the conversion from water to electricity. Hence, for expressing the problem in Spine format the following steps are taken for each power plant:

1) two nodes are introduced for modelling discharged and spilled water, one of them modelling $M_{i}$ (the upper node),

2) relationships are used to define the topology and the coupling of the decision variables,

3 ) one unit is introduced for modelling $P_{i, t}$, for each unit between a set of nodes three types of relationships are required, a set of relationships between each node and the unit that defines the topology, and a relationship coupling simultaneously all three entities that acts as a placeholder for the conversion ratio parameter,

4) two connections with the downstream power plant are used expressing $Q_{i, t}$ and $S_{i, t}$ respectively.

As shown in Fig. 3, two Spine nodes are used for each power plant (and therefore each hydrological balance constraint), an "upper" node and a "lower" node. In that way it is possible to emulate the diffluence of the two water flows - one corresponding to the water that is being spilled and hence not used for the electricity production, and the other one corresponding to the water that is being discharged for producing electricity. The connections are used for modelling the water flows between successive power plants. In conjunction with the upper and lower nodes setup, it can be observed that for each power plant there are at least two upstream and two downstream connections: one for spillage, connecting the upper node of a hydro power plant with the upper node of the next downstream power plant (i.e. water that is spilled and becomes available to the next plant), and the other one for the discharge, connecting the lower node of a plant with the upper node of the next plant (i.e. water that is used for electricity and can also be used by the next plant). The decision variables of the model accrue from the specification of nodes (e.g. the node state), units related to nodes (e.g. the flow between the unit and the node) and connections related to nodes (e.g. the flow between the connection and the node). It should be stressed that the links in Fig. 3 do not correspond to the actual water flows rather than define the coupling of the decision variables. By using these objects and relationships, it is possible to specify parameters that form the constraints, (such as the technical limits, the flow delays, etc.). Also, note that the "upper" and "lower" nodes of each power plant do not correspond to the level difference, but combined with the respective unit constitute one way for modelling the hydrobalance equation. The electricity production takes place between the "upper" node, the unit and the electricity node only. Lastly, the electricity 


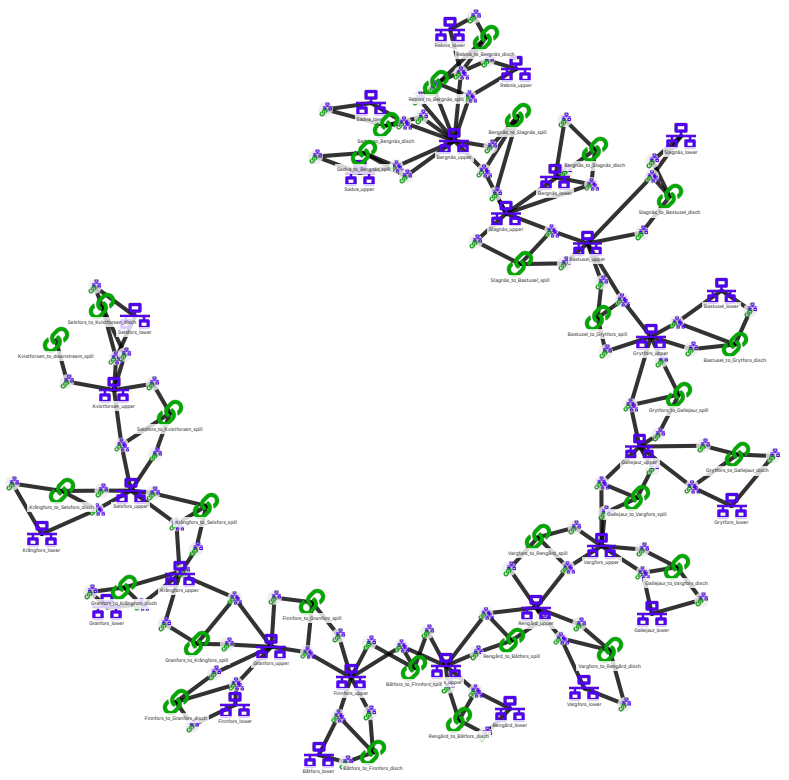

Fig. 4. Implementation of the Skellefte river system in Spine.

node only aggregates the electricity production of all power plants, imposing equation (1d), while implicitly defining the objective function being connected with an electricity load unit. In Fig. 4, the full implementation of the Skellefte river in Spine is presented (excluding units and commodities).

\section{RESUlts}

For assessing the practicality of using reduced resolutions in the hydro power model, we set about the simulation of the Skellefte river system for one week in an hourly basis. In Fig. 5 (a), the hourly electricity price timeseries for one week of operation is shown, that establishes the lowest possible time resolution for the hydro power model. By setting this resolution to the temporal block we simulate the system and extract some indicative results such as the total hourly discharge of the Sädva river (Fig. 5 (b)), and the total hourly discharge of the Gallejaur river (Fig. 5 (c)), but most importantly the total power produced by the river system shown in Fig. 5 (d). It is important to mention that the results can be easily extracted from, but also plotted by Spine toolbox.

As a next step, we evaluate additional simulation scenarios by gradually reducing the temporal resolution of the whole model (and therefore its complexity) and compare the results with the reference case that has the maximal time resolution. For scaling the model upwards we chose the calculation of the mean values for the redundant data. The comparison of simulation results with different resolutions is presented in Fig. 6 (a)-(c), while in Fig. 6 (d) we simulate a case where the time delays in the water flows have been disregarded (which also can be handled automatically by Spine). Although comparing the similarity of different time series data is a challenging and complex problem (especially for varying time resolution), and most importantly out of the scope of this work, it is still possible to draw conclusions regarding the (a)

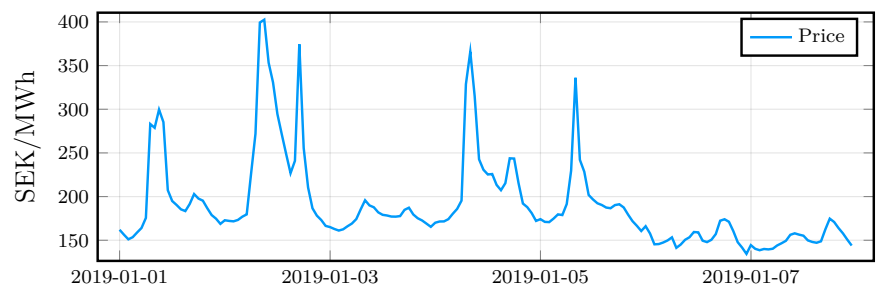

(b)

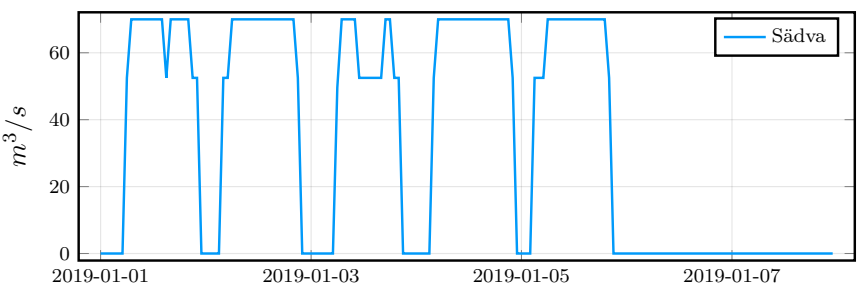

(c)

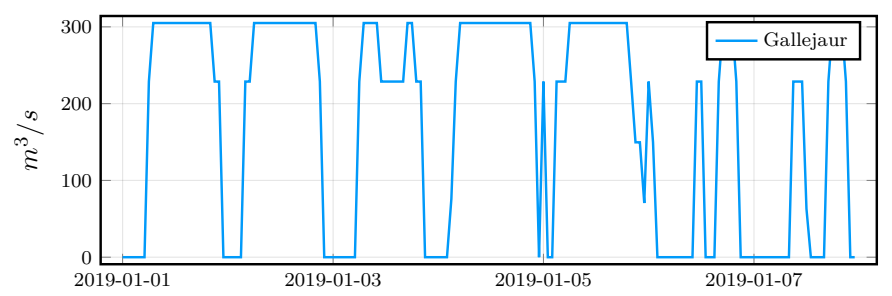

(d)

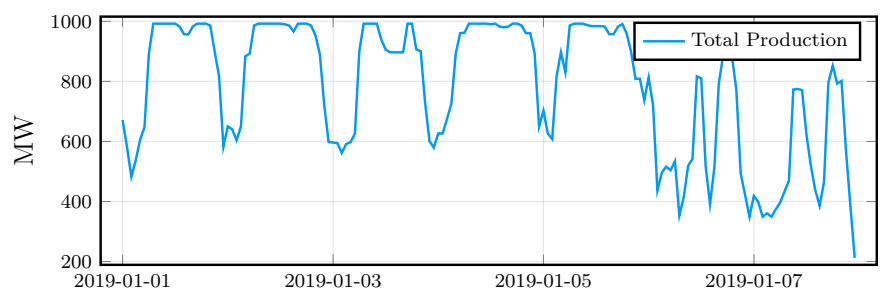

Fig. 5. Input parameters and indicative results from the hydro power model for one week, (a) electricity prices, (b) total hourly discharge of the Sädva river, (c) total hourly discharge of the Gallejaur river, (d) total power produced by the Skellefte river system.

efficacy of the models with reduced resolutions, by resorting to a qualitative evaluation, through the visual inspection of the results.

At the same time, we calculate and compare certain relevant indices for performing also a quantitative evaluation. In Table I, the error in the objective value disregarding the penalty factors (i.e. $\sum_{t=1}^{T} \lambda_{t} P_{t}$ ), and in the total energy that was produced (that is essentially the area defined by each curve) are shown for each case study. It is easy to observe that the similarity error indices in Table. I are maintained surprisingly low (accompanied by a very significant reduction in the complexity and model size indices), even for the 8hour resolution case that seems to deviate notably from the reference case. The exact opposite observation is made for the case where the time delays are disregarded. 
TABLE I

Similarity AND COMPUTATION COMPLEXITY STATISTICS FOR DifFERENT TEMPORAL RESOlutions.

\begin{tabular}{|c|c|c|c|c|c|c|}
\hline \multirow[t]{2}{*}{ Case study } & \multicolumn{2}{|c|}{ Similarity Error (\%) } & Computation Time & Total Memory & \multicolumn{2}{|c|}{ Model Statistics } \\
\hline & Objective Value & Total Production & \multicolumn{2}{|c|}{ Reduction (\%) } & Variables & Constraints \\
\hline Reference case & 26322450 & $131753 M W h$ & $19.189 s$ & $1.93 G B$ & 97104 & 182280 \\
\hline 2-hour resolution & 0.13 & 0.41 & 60.0 & 48.2 & 48552 & 91140 \\
\hline 4-hour resolution & 0.38 & 0.29 & 82.1 & 73.5 & 24276 & 45570 \\
\hline 8-hour resolution & 0.90 & 0.37 & 89.5 & 86.5 & 12138 & 22785 \\
\hline 12-hour resolution & 0.37 & 0.46 & 91.5 & 90.8 & 8092 & 15190 \\
\hline No delays & 3.97 & 4.9 & 30.2 & 0.52 & 97104 & 182280 \\
\hline
\end{tabular}

(a)

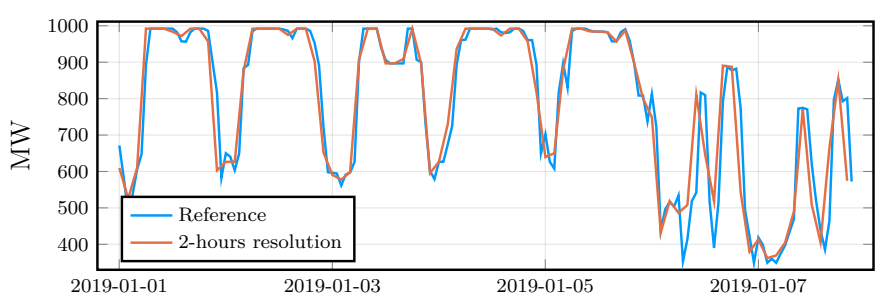

(b)

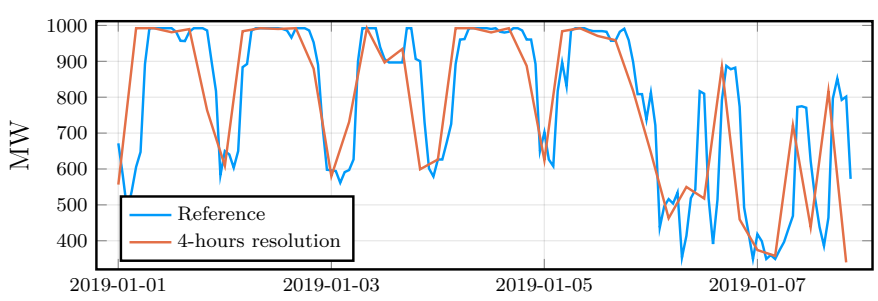

(c)

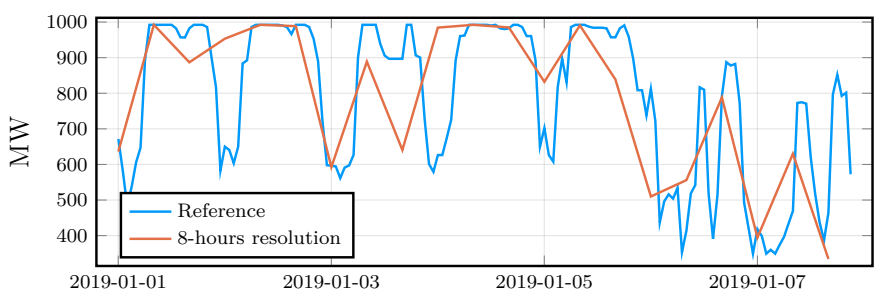

(d)

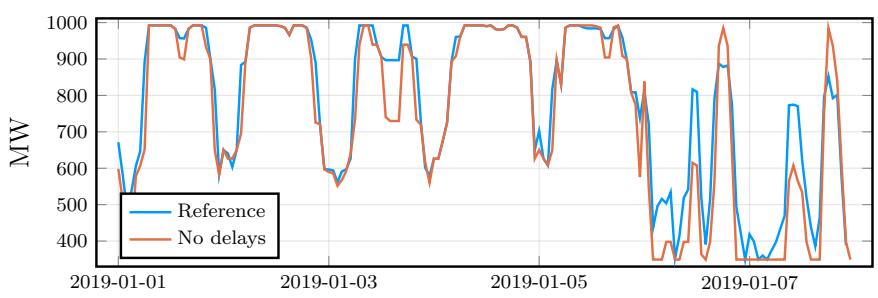

Fig. 6. Comparison of the total hourly electricity production with simulation scenarios of different temporal configurations, (a) 2-hour resolution, (b) 4hour resolution, (c) 8-hour resolution, (d) disregarding the time delays in the water flows.

\section{CONCLUSIONS}

In this paper we have shown the simulation of a specific river, although Spine has the possibility to simulate large power systems with hydro power being only a part of them. We have presented the modelling methodology and provided results from the hydro power system of the Skellefte river in Spine Model. By employing the automated model reduction capability of the Spine Model (in terms of reducing the temporal resolution), we showed that the computational complexity of the hydro power model can be drastically reduced, while it being able to provide meaningful results. In that way, the modeller can easily customize the granularity of the model according to the needs of the study. For the case of disregarding the water flow delays on the other hand, the results were significantly inferior.

\section{REFERENCES}

[1] G. J. Osório, J. C. O. Matias, and J. P. S. Catalão, "A review of shortterm hydro scheduling tools," in 2013 48th International Universities' Power Engineering Conference (UPEC), 2013, pp. 1-6.

[2] B. Wagner, C. Hauer, and H. Habersack, "Current hydropower developments in europe," Current Opinion in Environmental Sustainability, vol. 37 , pp. $41-49,2019$, sustainability challenges.

[3] F. Wiese, R. Bramstoft, H. Koduvere, A. P. Alonso], O. Balyk, J. G. Kirkerud, Åsa Grytli Tveten, T. F. Bolkesjø, M. Münster, and H. Ravn, "Balmorel open source energy system model," Energy Strategy Reviews, vol. 20, pp. $26-34,2018$.

[4] R. Brenes Brenes, "Hydropower modelling of continental europe using emps," Master's thesis, KTH, 2019, [Online]. Available: http://www.diva-portal.se/smash/get/diva2:1359928/FULLTEXT01.pdf.

[5] E. Blom, "Including hydropower in large scale power system models," KTH, Tech. Rep., 2019, [Online]. Available: http://kth.divaportal.org/smash/record.jsf?pid=diva2\%3A1320598\&dswid=3855.

[6] (2019) Project spine. [Online]. Available: http://www.spine-model.org/

[7] M. Marin, E. Rinne, P. Vennström, A. Soininen, and P. Savolainen. (2005) Spine toolbox user guide. [Online]. Available: https://spinetoolbox.readthedocs.io

[8] I. Dunning, J. Huchette, and M. Lubin, "Jump: A modeling language for mathematical optimization," SIAM Review, vol. 59, no. 2, pp. 295-320, 2017.

[9] K. Poncelet, H. Höschle, E. Delarue, A. Virag, and W. D'haeseleer, "Selecting representative days for capturing the implications of integrating intermittent renewables in generation expansion planning problems," IEEE Transactions on Power Systems, vol. 32, no. 3, pp. 1936-1948, 2017.

[10] D. Risberg and L. Söder, "Hydro power equivalents of complex river systems," in 2017 IEEE Manchester PowerTech, 2017, pp. 1-6.

[11] J. J. Sag, "Simulation of hydro power expansion in skellefteälven," Master's thesis, KTH, 2018, [Online]. Available: https://www.divaportal.org/smash/get/diva2:1215858/FULLTEXT02.

[12] L. Söder and M. Amelin, "Efficient operation and planning of power systems," 2011, qC 20111219. Course compendium, [Online]. Available: http://kth.divaportal.org/smash/record.jsf?pid=diva2\%3A467471\&dswid=-9693.

[13] E. Shayesteh, M. Amelin, and L. Söder, "Multi-station equivalents for short-term hydropower scheduling," IEEE Transactions on Power Systems, vol. 31, no. 6, pp. 4616-4625, 2016. 